\title{
Capturing Instance Level Ontology Evolution for DL-Lite
}

\author{
Evgeny Kharlamov and Dmitriy Zheleznyakov \\ KRDB Research Centre, Free University of Bozen-Bolzano, Italy \\ last_name@inf.unibz.it
}

\begin{abstract}
Evolution of Knowledge Bases (KBs) expressed in Description Logics (DLs) proved its importance. Recent studies of the topic mostly focussed on model-based approaches (MBAs), where an evolution (of a KB) results in a set of models. For KBs expressed in tractable DLs, such as DL-Lite, it was shown that the evolution suffers from inexpressibility, i.e., the result of evolution cannot be expressed in DL-Lite. What is missing in these studies is understanding: in which $D L$-Lite fragments evolution can be captured, what causes the inexpressibility, which logics is sufficient to express evolution, whether and how one can approximate it in DL-Lite. This work provides some understanding of these issues for eight of MBAs which cover the case of both update and revision. We found what causes inexpressibility and isolated a fragment of DL-Lite where evolution is expressible. For this fragment we provided polynomial-time algorithms to compute evolution results. For the general case we proposed techniques (based on what we called prototypes) to capture $D L$-Lite evolution corresponding to a well-known Winslett's approach in a DL $\mathcal{S H O I Q}$ (which is subsumed by OWL $2 \mathrm{DL}$ ). We also showed how to approximate this evolution in DL-Lite.
\end{abstract}

\section{Introduction}

Description Logics (DLs) provide excellent mechanisms for representing structured knowledge by means of Knowledge Bases (KBs) $\mathcal{K}$ that are composed of two components: TBox (describes intensional or general knowledge about an application domain) and ABox (describes facts about individual objects). DLs constitute the foundations for various dialects of OWL, the Semantic Web ontology language.

Traditionally DLs have been used for modeling static and structural aspects of application domains [1]. Recently, however, the scope of KBs has broadened, and they are now used also for providing support in the maintenance and evolution phase of information systems. This makes it necessary to study evolution of Knowledge Bases [2], where the goal is to incorporate new knowledge $\mathcal{N}$ into an existing KB $\mathcal{K}$ so as to take into account changes that occur in the underlying application domain. In general, $\mathcal{N}$ is represented by a set of formulas denoting properties that should be true after $\mathcal{K}$ has evolved, and the result of evolution, denoted $\mathcal{K} \diamond \mathcal{N}$, is also intended to be a set of formulas. In the case where $\mathcal{N}$ interacts with $\mathcal{K}$ in an undesirable way, e.g., by causing the $\mathrm{KB}$ or relevant parts of it to become unsatisfiable, $\mathcal{N}$ cannot be simply added to the KB. Instead, suitable changes need to be made in $\mathcal{K}$ so as to avoid this undesirable interaction, e.g., by deleting parts of $\mathcal{K}$ conflicting with $\mathcal{N}$. Different choices for changes are possible, corresponding to different approaches to semantics for KB evolution [3|4|5]. 
An important group of approaches to evolution semantics, that we focus in this paper, is called model-based (MBAs). Under MBAs the result of evolution $\mathcal{K} \diamond \mathcal{N}$ is a set of models of $\mathcal{N}$ that are minimally distanced from models of $\mathcal{K}$. Depending on what the distance between models is and how to measure it, eight different MBAs were introduced (see Section 2.2 for details). Since $\mathcal{K} \diamond \mathcal{N}$ is a set of models, while $\mathcal{K}$ and $\mathcal{N}$ are logical theories, it is desirable to represent $\mathcal{K} \diamond \mathcal{N}$ as a logical theory using the same language as for $\mathcal{K}$ and $\mathcal{N}$. Thus, looking for representations of $\mathcal{K} \diamond \mathcal{N}$ is the main challenge in studies of evolution under MBAs. When $\mathcal{K}$ and $\mathcal{N}$ are propositional theories, representing $\mathcal{K} \diamond \mathcal{N}$ is well understood [5], while it becomes dramatically more complicated as soon as $\mathcal{K}$ and $\mathcal{N}$ are first-order, e.g., DL KBs [6].

Model based evolution of KBs where $\mathcal{K}$ and $\mathcal{N}$ are written in a language of the $D L$-Lite family [7] has been recently extensively studied [689]. The focus on $D L$ Lite is not surprising since it is the basis of OWL $2 \mathrm{QL}$, a tractable OWL 2 profile. It has been shown that for every of the eight MBAs one can find DL-Lite $\mathcal{K}$ and $\mathcal{N}$ such that $\mathcal{K} \diamond \mathcal{N}$ cannot be expressed in DL-Lite [1011], i.e., DL-Lite is not closed under MBA evolution. This phenomenon was also noted in [6 10] for some of the eight semantics. What is missing in all these studies of evolution for DL-Lite is understanding of

(1) DL-Lite wrt evolution: What DL-Lite fragments are closed under MBAs? What $D L$ Lite formulas are in charge of inexpressibility?

(2) Evolution wrt DL-Lite : Is it possible and how to capture evolution of DL-Lite KBs in richer logics? What are these logics?

(3) Approximation of evolution results: For DL-Lite $\mathrm{KB} \mathcal{K}$ and an $\mathrm{ABox} \mathcal{N}$, is it possible and how to do "good" approximations of $\mathcal{K} \diamond \mathcal{N}$ in DL-Lite?

In this paper we study the problems (1)-(3) for so-called ABox evolution, i.e., $\mathcal{N}$ is a new ABox and the TBox of $\mathcal{K}$ should remain the same after the evolution. ABox evolution is important for areas, e.g., artifact-centered service interoperation (http: / / www . acsi-project.eu/), where the structural knowledge (TBox) is well crafted and stable, while (ABox) facts about individuals may get changed. These ABox changes should be reflected in KBs in a way that the TBox is not affected. Our study covers both the case of ABox updates and ABox revision [4].

The contributions of the paper are: We provide relationships between MBAs for $D L-$ Lite $_{\mathcal{R}}$ by showing which approaches subsume each other (Section 3 ). We introduce $D L$-Lite $p_{\mathcal{R}}^{p r}$, a restriction on $D L$-Lite $\mathcal{R}_{\mathcal{R}}$ where disjointness of concepts with role projections is forbidden. We show that $D L-L_{\text {Lite }} e_{\mathcal{R}}^{p r}$ is closed under most of MBA evolutions and provide polynomial-time algorithms to compute (representations of) $\mathcal{K} \diamond \mathcal{N}$ (Section 4). For DL- Lite $_{\mathcal{R}}$ we focus on an important MBA corresponding to a well accepted Winslett's semantics and show how to capture $\mathcal{K} \diamond \mathcal{N}$ for this semantics in a DL $\mathcal{S H O I Q}$ (Section 5). We show what combination of assertions in $\mathcal{T}$ together with $\mathcal{N}$ can lead to inexpressibility of $(\mathcal{T}, \mathcal{A}) \diamond \mathcal{N}$ in $D$ L-Lite $\mathcal{R}_{\mathcal{R}}$ (Section5.1). For the case when $\mathcal{K} \diamond \mathcal{N}$ is not expressible in $D$ L-Lite L $_{\mathcal{R}}$ we study how to approximate it in DL-Lite $\mathcal{R}_{\mathcal{R}}$ (Section 5.4). 


\section{Preliminaries}

\subsection{DL-Lite $_{\mathcal{R}}$}

We introduce some basic notions of DLs, (see [1] for more details). We consider a logic DL-Lite $\mathcal{R}_{\mathcal{R}}$ of DL-Lite family of DLs [7]12]. DL-Lite $\mathcal{R}_{\mathcal{R}}$ has the following constructs for (complex) concepts and roles: (i) $B::=A \mid \exists R$, (ii) $C::=B \mid \neg B$, (iii) $R::=P \mid P^{-}$, where $A$ and $P$ stand for an atomic concept and role, respectively, which are just names. A knowledge base $(\mathrm{KB}) \mathcal{K}=(\mathcal{T}, \mathcal{A})$ is compounded of two sets of assertions: TBox $\mathcal{T}$, and ABox $\mathcal{A}$. DL-Lite $\mathcal{R}_{\mathcal{R}}$ TBox assertions are concept inclusion assertions of the form $B \sqsubseteq C$ and role inclusion assertions $R_{1} \sqsubseteq R_{2}$, while $\mathrm{ABox}$ assertions are membership assertions of the form $A(a), \neg A(a)$, and $R(a, b)$. The active domain of $\mathcal{K}$, denoted $\operatorname{adom}(\mathcal{K})$, is the set of all constants occurring in $\mathcal{K}$. In Section 5

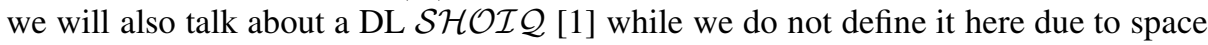
limit.

The semantics of DL-Lite KBs is given in the standard way, using first order interpretations, all over the same countable domain $\Delta$. An interpretation $\mathcal{I}$ is a function.$^{\mathcal{I}}$ that assigns to each $C$ a subset $C^{\mathcal{I}}$ of $\Delta$, and to $R$ a binary relation $R^{\mathcal{I}}$ over $\Delta$ in a way that $(\neg B)^{\mathcal{I}}=\Delta \backslash B^{\mathcal{I}},(\exists R)^{\mathcal{I}}=\left\{a \mid \exists a^{\prime} .\left(a, a^{\prime}\right) \in R^{\mathcal{I}}\right\}$, and $\left(P^{-}\right)^{\mathcal{I}}=$ $\left\{\left(a_{2}, a_{1}\right) \mid\left(a_{1}, a_{2}\right) \in P^{\mathcal{I}}\right\}$. We assume that $\Delta$ contains the constants and that $c^{\mathcal{I}}=c$ (we adopt standard names). Alternatively, we view interpretations as sets of atoms and say that $A(a) \in \mathcal{I}$ iff $a \in A^{\mathcal{I}}$ and $P(a, b) \in \mathcal{I}$ iff $(a, b) \in P^{\mathcal{I}}$. An interpretation $\mathcal{I}$ is a model of a membership assertion $A(a)$ (resp., $\neg A(a)$ ) if $a \in A^{\mathcal{I}}$ (resp., $a \notin A^{\mathcal{I}}$ ), of $P(a, b)$ if $(a, b) \in P^{\mathcal{I}}$, and of an assertion $D_{1} \sqsubseteq D_{2}$ if $D_{1}^{\mathcal{I}} \subseteq$ $D_{2}^{\mathcal{I}}$.

As usual, we use $\mathcal{I} \models F$ to denote that $\mathcal{I}$ is a model of an assertion $F$, and $\mathcal{I} \models \mathcal{K}$ denotes that $\mathcal{I} \models F$ for each $F$ in $\mathcal{K}$. We use $\operatorname{Mod}(\mathcal{K})$ to denote the set of all models of $\mathcal{K}$. A KB is satisfiable if it has at least one model. The $D L$-Lite family has nice computational properties, for example, KB satisfiability has polynomial-time complexity in the size of the TBox and logarithmic-space in the size of the ABox [12[13]. We use entailment on KBs $\mathcal{K} \models \mathcal{K}^{\prime}$ in the standard sense. An ABox $\mathcal{A} \mathcal{T}$-entails an ABox $\mathcal{A}^{\prime}$, denoted $\mathcal{A} \models_{\mathcal{T}} \mathcal{A}^{\prime}$, if $\mathcal{T} \cup \mathcal{A} \models \mathcal{A}^{\prime}$, and $\mathcal{A}$ is $\mathcal{T}$-equivalent to $\mathcal{A}^{\prime}$, denoted $\mathcal{A} \equiv_{\mathcal{T}} \mathcal{A}^{\prime}$, if $\mathcal{A} \models_{\mathcal{T}} \mathcal{A}^{\prime}$ and $\mathcal{A}^{\prime} \models_{\mathcal{T}} \mathcal{A}$.

The deductive closure of a TBox $\mathcal{T}$, denoted $\operatorname{cl}(\mathcal{T})$, is the set of all TBox assertions $F$ such that $\mathcal{T}=F$. For satisfiable $\operatorname{KBs} \mathcal{K}=(\mathcal{T}, \mathcal{A}$ ), a full closure of $\mathcal{A}$ (wrt $\mathcal{T}$ ), denoted $f c_{\mathcal{T}}(\mathcal{A})$, is the set of all membership assertions $f$ (both positive and negative) over $\operatorname{adom}(\mathcal{K})$ such that $\mathcal{A} \models_{\mathcal{T}} f$. In $D L$-Lite $e_{\mathcal{R}}$ both $c l(\mathcal{T})$ and $f c l_{\mathcal{T}}(\mathcal{A})$ are computable in time quadratic in, respectively, $|\mathcal{T}|$, i.e., the number of assertions of $\mathcal{T}$, and $\mid \mathcal{T} \cup$ $\mathcal{A} \mid$. In our work we assume that all TBoxes and ABoxes are closed, while results are extendable to arbitrarily KBs.

A homomorphism $h$ from a model $\mathcal{I}$ to a model $\mathcal{J}$ is a mapping from $\Delta$ to $\Delta$ satisfying: (i) $h(a)=a$ for every constant $a$; (ii) if $\alpha \in A^{\mathcal{I}}$ (resp., $(\alpha, \beta) \in P^{\mathcal{I}}$ ), then $h(\alpha) \in A^{\mathcal{J}}$ (resp., $(h(\alpha), h(\beta)) \in P^{\mathcal{J}}$ ) for every $A$ (resp., $P$ ). A canonical model of $\mathcal{K}$ is a model which can be homomorphically embedded in every model of $\mathcal{K}$, denoted $\mathcal{I}_{\mathcal{K}}^{\text {can }}$ or just $\mathcal{I}^{\text {can }}$ when $\mathcal{K}$ is clear from the context. 

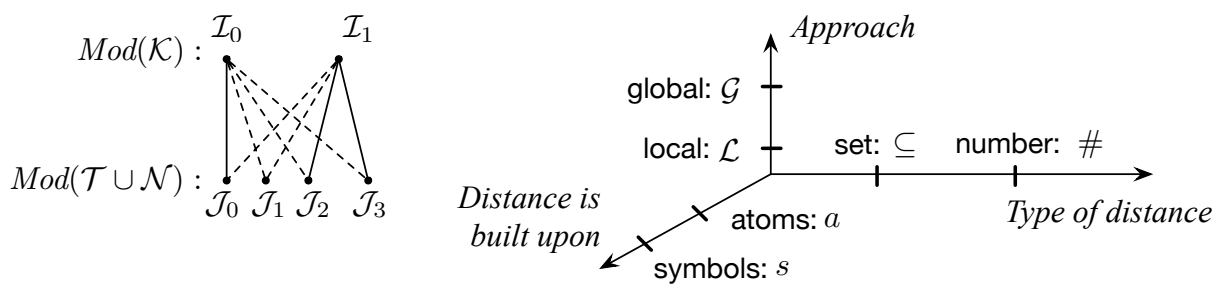

Fig. 1. Left: measuring distances between models and finding local minimums.

Right: three-dimensional space of approaches to model-based evolution semantics.

\subsection{Evolution of Knowledge Bases}

This section is based on [10]. Let $\mathcal{K}=(\mathcal{T}, \mathcal{A})$ be a $D L$-Lite $_{\mathcal{R}} \mathrm{KB}$ and $\mathcal{N}$ a "new" ABox. We study how to incorporate $\mathcal{N}$ 's assertions into $\mathcal{K}$, that is, how $\mathcal{K}$ evolves under $\mathcal{N}$ [2]. More practically, we study evolution operators that take $\mathcal{K}$ and $\mathcal{N}$ as input and return, possibly in polynomial time, a $D L$-Lite $_{\mathcal{R}} \mathcal{K}^{\prime}=\left(\mathcal{T}, \mathcal{A}^{\prime}\right)$ (with the same TBox as $\mathcal{K}$ ) that captures the evolution, and which we call the $(A B o x)$ evolution of $\mathcal{K}$ under $\mathcal{N}$. Based on the evolution principles of [10], we require $\mathcal{K}$ and $\mathcal{K}^{\prime}$ to be satisfiable. A $D L$-Lite $\mathcal{R}$ $\mathrm{KB} \mathcal{K}=(\mathcal{T}, \mathcal{A})$ and an $\mathrm{ABox} \mathcal{N}$ is a evolution setting if $\mathcal{K}$ and $(\mathcal{T}, \mathcal{N})$ are satisfiable.

Model-Based Semantics of Evolution. In model-based approaches (MBAs), the result of evolution of a $\mathrm{KB} \mathcal{K}$ wrt new knowledge $\mathcal{N}$ is a set $\mathcal{K} \diamond \mathcal{N}$ of models. The idea of MBAs is to choose as $\mathcal{K} \diamond \mathcal{N}$ some models of $(\mathcal{T}, \mathcal{N})$ depending on their distance to $\mathcal{K}$ 's models. Katsuno and Mendelzon [4] considered two ways, so called local and global, of choosing these models of $(\mathcal{T}, \mathcal{N})$, where the first choice corresponds to knowledge update and the second one to knowledge revision.

The idea of the local approaches is to consider all models $\mathcal{I}$ of $\mathcal{K}$ one by one and for each $\mathcal{I}$ to take those models $\mathcal{J}$ of $(\mathcal{T}, \mathcal{N})$ that are minimally distant from $\mathcal{I}$. Formally,

$$
\mathcal{K} \diamond \mathcal{N}=\bigcup_{\mathcal{I} \in \operatorname{Mod}(\mathcal{K})} \mathcal{I} \diamond \mathcal{N}, \text { where } \mathcal{I} \diamond \mathcal{N}=\underset{\mathcal{J} \in \operatorname{Mod}(\mathcal{T} \cup \mathcal{N})}{\arg \min } \operatorname{dist}(\mathcal{I}, \mathcal{J}) .
$$

where $\operatorname{dist}(\cdot, \cdot)$ is a function whose range is a partially ordered domain and arg min stands for the argument of the minimum, that is, in our case, the set of models $\mathcal{J}$ for which the value of $\operatorname{dist}(\mathcal{I}, \mathcal{J})$ reaches its minimum value, given $\mathcal{I}$. The distance function dist varies from approach to approach and commonly takes as values either numbers or subsets of some fixed set. To get a better intuition of the local semantics, consider Figure 1, left, where we present two model $\mathcal{I}_{0}$ and $\mathcal{I}_{1}$ of a $\mathrm{KB} \mathcal{K}$ and four models $\mathcal{J}_{0}, \ldots, \mathcal{J}_{3}$ of $(\mathcal{T}, \mathcal{N})$. We represent the distance between a model of $\mathcal{K}$ and a model of $\mathcal{T} \cup \mathcal{N}$ by the length of a line connecting them. Solid lines correspond to minimal distances, dashed lines to distances that are not minimal. In this figure $\left\{\mathcal{J}_{0}\right\}=$ $\arg \min _{\mathcal{J} \in\left\{\mathcal{J}_{0}, \ldots, \mathcal{J}_{3}\right\}} \operatorname{dist}\left(\mathcal{I}_{0}, \mathcal{J}\right)$ and $\left\{\mathcal{J}_{2}, \mathcal{J}_{3}\right\}=\arg \min _{\mathcal{J} \in\left\{\mathcal{J}_{0}, \ldots, \mathcal{J}_{3}\right\}} \operatorname{dist}\left(\mathcal{I}_{1}, \mathcal{J}\right)$.

In the global approach one choses models of $\mathcal{T} \cup \mathcal{N}$ that are minimally distant from $\mathcal{K}:$

$$
\mathcal{K} \diamond \mathcal{N}=\underset{\mathcal{J} \in \operatorname{Mod}(\mathcal{T} \cup \mathcal{N})}{\arg \min } \operatorname{dist}(\operatorname{Mod}(\mathcal{K}), \mathcal{J})
$$


where $\operatorname{dist}(\operatorname{Mod}(\mathcal{K}), \mathcal{J})=\min _{\mathcal{I} \in \operatorname{Mod}(\mathcal{K})} \operatorname{dist}(\mathcal{I}, \mathcal{J})$. Consider again Figure 1 left, and assume that the distance between $\mathcal{I}_{0}$ and $\mathcal{J}_{0}$ is the global minimum, hence, $\left\{\mathcal{J}_{0}\right\}=$ $\arg \min _{\mathcal{J} \in\left\{\mathcal{J}_{0}, \ldots, \mathcal{J}_{3}\right\}} \operatorname{dist}\left(\left\{\mathcal{I}_{0}, \mathcal{I}_{1}\right\}, \mathcal{J}\right)$.

Measuring Distance Between Interpretations. The classical MBAs were developed for propositional theories [5], where interpretation were sets of propositional atoms, two distance functions were introduced, respectively based on symmetric difference " $\ominus$ " and on the cardinality of symmetric difference:

$$
\operatorname{dist}_{\subseteq}(\mathcal{I}, \mathcal{J})=\mathcal{I} \ominus \mathcal{J} \quad \text { and } \quad \operatorname{dist}_{\#}(\mathcal{I}, \mathcal{J})=|\mathcal{I} \ominus \mathcal{J}|,
$$

where $\mathcal{I} \ominus \mathcal{J}=(\mathcal{I} \backslash \mathcal{J}) \cup(\mathcal{J} \backslash \mathcal{I})$. Distances under dist $\subseteq$ are sets and are compared by set inclusion, that is, dist $_{\subseteq}\left(\mathcal{I}_{1}, \mathcal{J}_{1}\right) \leq \operatorname{dist}_{\subseteq}\left(\mathcal{I}_{2}, \mathcal{J}_{2}\right)$ iff $\overline{d i s t}_{\subseteq}\left(\mathcal{I}_{1}, \mathcal{J}_{1}\right) \subseteq \operatorname{dist}_{\subseteq}\left(\mathcal{I}_{2}, \mathcal{J}_{2}\right)$. Finite distances under dist $\#$ are natural numbers and are compared in the standard way.

One can extend these distances to DL interpretations in two different ways. One way is to consider interpretations $\mathcal{I}, \mathcal{J}$ as sets of atoms. Then $\mathcal{I} \ominus \mathcal{J}$ is again a set of atoms and we can define distances as in Equation (2). We denote these distances as $\operatorname{dist}_{\subseteq}^{a}(\mathcal{I}, \mathcal{J})$ and $\operatorname{dist}_{\#}^{a}(\mathcal{I}, \mathcal{J})$. Another way is to define distances at the level of the concept and role symbols in the signature $\Sigma$ underlying the interpretations:

$$
\operatorname{dist}_{\subseteq}^{s}(\mathcal{I}, \mathcal{J})=\left\{S \in \Sigma \mid S^{\mathcal{I}} \neq S^{\mathcal{J}}\right\}, \quad \text { and } \quad \operatorname{dist}_{\#}^{s}(\mathcal{I}, \mathcal{J})=\left|\left\{S \in \Sigma \mid S^{\mathcal{I}} \neq S^{\mathcal{J}}\right\}\right| .
$$

Summing up across the different possibilities, we have three dimensions, which give eight semantics of evolution according to MBAs by choosing: (1) the local or the global approach, (2) atoms or symbols for defining distances, and (3) set inclusion or cardinality to compare symmetric differences. In Figure 1, right, we depict these three dimensions. We denote each of these eight possibilities by a combination of three symbols, indicating the choice in each dimension, e.g., $\mathcal{L}_{\#}^{a}$ denotes the local semantics where the distances are expressed in terms of cardinality of sets of atoms.

Closure Under Evolution. Let $\mathcal{D}$ be a DL and $M$ one of the eight MBAs introduced above. We say $\mathcal{D}$ is closed under evolution wrt $M$ (or evolution wrt $M$ is expressible in $\mathcal{D}$ ) if for every evolution setting $\mathcal{K}$ and $\mathcal{N}$ written in $\mathcal{D}$, there is a $\mathrm{KB} \mathcal{K}^{\prime}$ written in $\mathcal{D}$ such that $\operatorname{Mod}\left(\mathcal{K}^{\prime}\right)=\mathcal{K} \diamond \mathcal{N}$, where $\mathcal{K} \diamond \mathcal{N}$ is the evolution result under semantics $M$.

We showed in [10[11] that DL-Lite is not closed under any of the eight model based semantics. The observation underlying these results is that on the one hand, the minimality of change principle intrinsically introduces implicit disjunction in the evolved KB. On the other hand, since DL-Lite is a slight extension of Horn logic [14], it does not allow one to express genuine disjunction (see Lemma 1 in [10] for details).

Let $M$ be a set of models that resulted from the evolution of $(\mathcal{T}, \mathcal{A})$ with $\mathcal{N}$. A KB $\left(\mathcal{T}, \mathcal{A}^{\prime}\right)$ is a sound approximation of $M$ if $M \subseteq \operatorname{Mod}\left(\mathcal{T}, \mathcal{A}^{\prime}\right)$. A sound approximation $\left(\mathcal{T}, \mathcal{A}^{\prime}\right)$ is minimal if for every sound approximation $\left(\mathcal{T}, \mathcal{A}^{\prime \prime}\right)$ inequivalent to $\left(\mathcal{T}, \mathcal{A}^{\prime}\right)$, it holds that $\operatorname{Mod}\left(\mathcal{T}, \mathcal{A}^{\prime \prime}\right) \not \subset \operatorname{Mod}\left(\mathcal{T}, \mathcal{A}^{\prime}\right)$, i.e., $\left(\mathcal{T}, \mathcal{A}^{\prime}\right)$ is minimal wrt " $\subseteq$ ".

\section{Relationships between Model-Based Semantics}

Let $\mathcal{S}_{1}$ and $\mathcal{S}_{2}$ be two evolution semantics and $\mathcal{D}$ a logic language. Then $\mathcal{S}_{1}$ is subsumed by $\mathcal{S}_{2}$ wrt $\mathcal{D}$, denoted $\left(\mathcal{S}_{1} \preccurlyeq_{\text {sem }} \mathcal{S}_{2}\right)(\mathcal{D})$, or just $\mathcal{S}_{1} \preccurlyeq_{\text {sem }} \mathcal{S}_{2}$ when $\mathcal{D}$ is clear from the 


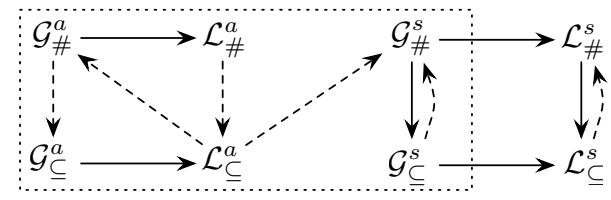

Fig. 2. Subsumptions for evolution semantics.

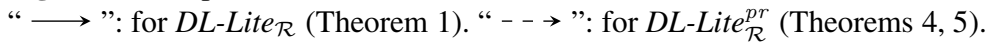

Dashed frame surrounds semantics under which $D L-L i t e_{\mathcal{R}}^{p r}$ is closed.

context, if $\mathcal{K} \diamond_{\mathcal{S}_{1}} \mathcal{N} \subseteq \mathcal{K} \diamond_{\mathcal{S}_{2}} \mathcal{N}$ for all satisfiable KBs $\mathcal{K}$ and $\mathcal{N}$ written in $\mathcal{D}$, where $\mathcal{K} \diamond_{\mathcal{S}_{i}} \mathcal{N}$ denotes evolution under $\mathcal{S}_{i}$. Two semantics $\mathcal{S}_{1}$ and $\mathcal{S}_{2}$ are equivalent (wrt $\mathcal{D})$, denoted $\left(\mathcal{S}_{1} \equiv_{\text {sem }} \mathcal{S}_{2}\right)(\mathcal{D})$, if $\left(\mathcal{S}_{1} \preccurlyeq_{\text {sem }} \mathcal{S}_{2}\right)(\mathcal{D})$ and $\left(\mathcal{S}_{2} \preccurlyeq\right.$ sem $\left.\mathcal{S}_{1}\right)(\mathcal{D})$. Further in this section we will consider $\mathcal{K}$ and $\mathcal{N}$ written in $D L$-Lite $\mathcal{R}_{\mathcal{R}}$. The following theorem shows the subsumption relation between different semantics. We depict these relations in Figure 2 using solid arrows. The figure is complete in the following sense: there is a solid path (a sequence of solid arrows) between any two semantics $\mathcal{S}_{1}$ and $\mathcal{S}_{2}$ iff there is a subsumption $\mathcal{S}_{1} \preccurlyeq$ sem $\mathcal{S}_{2}$.

Theorem 1. Let $\beta \in\{a, s\}$ and $\alpha \in\{\subseteq, \#\}$. Then for DL-Lite $\mathcal{R}_{\mathcal{R}}$ it holds that

$$
\mathcal{G}_{\alpha}^{\beta} \preccurlyeq{ }_{\text {sem }} \mathcal{L}_{\alpha}^{\beta}, \quad \mathcal{G}_{\#}^{s} \preccurlyeq_{\text {sem }} \mathcal{G}_{\subseteq}^{s}, \quad \text { and } \quad \mathcal{L}_{\#}^{s} \preccurlyeq_{\text {sem }} \mathcal{L}_{\subseteq}^{s} .
$$

Proof. Let dist ${ }_{\alpha}^{\beta}$ be a distance function, $\mathcal{E}_{\mathcal{G}}=\mathcal{K} \diamond \mathcal{N}$ wrt $\mathcal{G}_{\alpha}^{\beta}$ and $\mathcal{E}_{\mathcal{L}}=\mathcal{K} \diamond \mathcal{N}$ wrt $\mathcal{L}_{\alpha}^{\beta}$ be corresponding global and local semantics based on dist ${ }_{\alpha}^{\beta}$. For an evolution setting $\mathcal{K}$ and $\mathcal{N}$, let $\mathcal{J}^{\prime} \in \mathcal{E}_{\mathcal{G}}$. Then, there is $\mathcal{I}^{\prime} \models \mathcal{K}$ such that for every $\mathcal{I}^{\prime \prime} \models \mathcal{K}$ and $\mathcal{J}^{\prime \prime}=\mathcal{T} \cup \mathcal{N}$ it does not hold that $\operatorname{dist}_{\alpha}^{\beta}\left(\mathcal{I}^{\prime \prime}, \mathcal{J}^{\prime \prime}\right) \lesseqgtr \operatorname{dist}_{\alpha}^{\beta}\left(\mathcal{I}^{\prime}, \mathcal{J}^{\prime}\right)$. In particular, when $\mathcal{I}^{\prime \prime}=\mathcal{I}^{\prime}$, there is no $\mathcal{J}^{\prime \prime} \models \mathcal{T} \cup \mathcal{N}$ such that $\operatorname{dist}_{\alpha}^{\beta}\left(\mathcal{I}^{\prime}, \mathcal{J}^{\prime \prime}\right) \lesseqgtr \operatorname{dist}_{\alpha}^{\beta}\left(\mathcal{I}^{\prime}, \mathcal{J}^{\prime}\right)$, which yields that $\mathcal{J}^{\prime} \in \arg \min _{\mathcal{J} \in \operatorname{Mod}(\mathcal{T} \cup \mathcal{N})} \operatorname{dist}_{\alpha}^{\beta}\left(\mathcal{I}^{\prime}, \mathcal{J}\right)$, and $\mathcal{J}^{\prime} \in \mathcal{E}_{\mathcal{L}}$. We conclude that: $\mathcal{G}_{\#}^{a} \preccurlyeq$ sem $\mathcal{L}_{\#}^{a}, \quad \mathcal{G}_{\subseteq}^{a} \preccurlyeq$ sem $\mathcal{L}_{\subseteq}^{a}, \quad \mathcal{G}_{\#}^{s} \preccurlyeq_{\text {sem }} \mathcal{L}_{\#}^{s}, \quad \mathcal{G}_{\subseteq}^{s} \preccurlyeq_{\text {sem }} \mathcal{L}_{\subseteq}^{s}$.

Consider $\mathcal{E}_{\#}=\mathcal{K} \diamond \mathcal{N}$ wrt $\mathcal{L}_{\#}^{\beta}$, which is based on dist $_{\#}$, and $\mathcal{E}_{\subseteq}=\mathcal{K} \diamond \mathcal{N}$ wrt $\mathcal{L}_{\subseteq}^{\beta}$, which is based on dist $_{\subseteq}$. We now check whether $\mathcal{E}_{\#} \preccurlyeq$ sem $\mathcal{E}_{\subseteq}$ holds. Assume $\mathcal{J}^{\prime} \in \mathcal{E}_{\#}$ and $\mathcal{J}^{\prime} \notin \mathcal{E}_{\subseteq}$. Then, from the former assumption we conclude existence of $\mathcal{I}^{\prime} \models \mathcal{K}$ such that $\mathcal{J}^{\prime} \in \arg \min \mathcal{J} \in \operatorname{Mod}(\mathcal{T} \cup \mathcal{N})_{\text {dist }}\left(\mathcal{I}^{\prime}, \mathcal{J}\right)$. From the latter assumption, $\mathcal{J}^{\prime} \notin \mathcal{E}_{\subseteq}$, we conclude existence of a model $\mathcal{J}^{\prime \prime}$ such that $\operatorname{dist}_{\subseteq}\left(\mathcal{I}^{\prime}, \mathcal{J}^{\prime \prime}\right) \subsetneq \operatorname{dist} \subseteq\left(\mathcal{I}^{\prime}, \mathcal{J}^{\prime}\right)$. This yields that $\operatorname{dist}_{\#}\left(\mathcal{I}^{\prime}, \mathcal{J}^{\prime \prime}\right) \lesseqgtr \operatorname{dist}_{\#}\left(\mathcal{I}^{\prime}, \mathcal{J}^{\prime}\right)$, which contradicts the fact that $\mathcal{J}^{\prime} \in \mathcal{E}_{\#}$, assuming that $\operatorname{dist}_{\subseteq}\left(\mathcal{I}^{\prime}, \mathcal{J}^{\prime}\right)$ is finite. Thus, $\mathcal{E}_{\#} \preccurlyeq$ sem $\mathcal{E}_{\subseteq}$ as soon as $\operatorname{dist}_{\subseteq}(\mathcal{I}, \mathcal{J})$ is finite. This finiteness condition always holds for when $\beta=s$ since the signature of $\mathcal{K} \cup \mathcal{N}$ is finite. It is easy to check that $\operatorname{dist}_{\subseteq}(\mathcal{I}, \mathcal{J})$ may not be finite when $\beta=a$, hence, $\mathcal{L}_{\#}^{a} \npreceq_{\text {sem }} \mathcal{L}_{\subseteq}^{a}$.

Similarly, one can show that $\mathcal{G}_{\#}^{s} \preccurlyeq_{\text {sem }} \mathcal{G}_{\subseteq}^{s}$ and $\mathcal{G}_{\#}^{a} \aleph_{\text {sem }} \mathcal{G}_{\subseteq}^{a}$ in $D L$-Lite $\mathcal{R}$.

\section{Evolution of $D L$-Lite ${ }_{\mathcal{R}}^{p r} \mathrm{KBS}$}

Consider a restriction of $D L$-Lite $\mathcal{R}_{\mathcal{R}}$, which we call $D L$-Lite $e_{\mathcal{R}}^{p r}$ ( $p r$ stands for positive role interaction), where disjointness that involves roles is forbidden (only positive inclusions 


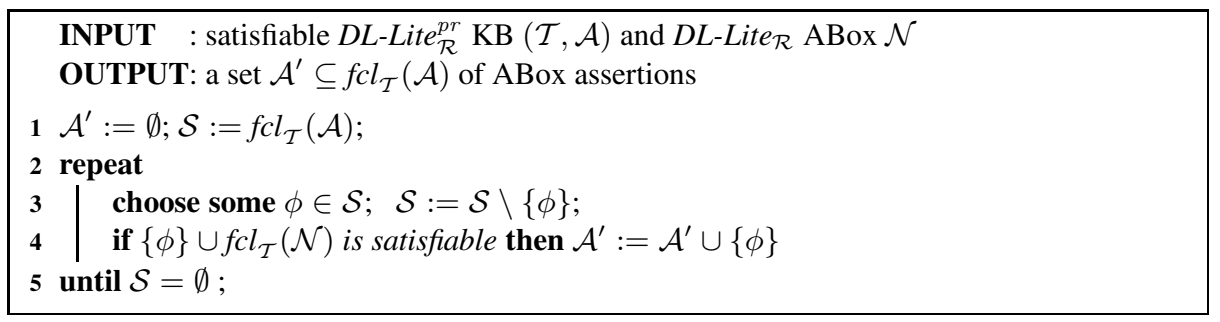

Algorithm 1. Algorithm $\operatorname{Align} \operatorname{Alg}((\mathcal{T}, \mathcal{A}), \mathcal{N})$ for $\mathcal{A}^{\prime}$ deterministic computation

involving roles are permitted). Formally, $\mathcal{T}$ is in $D L-L i t e_{\mathcal{R}}^{p r}$ if it is in DL-Lite $\mathcal{R}_{\mathcal{R}}$ and $\mathcal{T} \forall$ $\exists R \sqsubseteq \neg B$ for any role $R$ and any concept $B$. DL-Lite $e_{\mathcal{R}}^{p r}$ is defined semantically, while one can syntactically check (in quadratic time), given a $D L$-Lite $e_{\mathcal{R}}$ TBox $\mathcal{T}$ whether it is in $D L$-Lite $e_{\mathcal{R}}^{p r}$ : compute a closure of $\mathcal{T}$, check that no assertion of the form $\exists R \sqsubseteq \neg B$ is in the closure and if it is the case, then $\mathcal{K}$ is in $D L-L i t e_{\mathcal{R}}^{p r}$. Note that DL-Lite $p_{\mathcal{R}}^{p r}$ is an extension of RDFS ontology language (of its first-order logics fragment). DL-Lite $e_{\mathcal{R}}^{p r}$ adds to RDFS the ability of expressing disjointness of atomic concepts $\left(A_{1} \sqsubseteq \neg A_{2}\right)$ and mandatory participation ( $A \sqsubseteq \exists R$ ). In the rest of the section we investigate whether and how to capture $\mathcal{K} \diamond \mathcal{N}$ in $D L-L i t e_{\mathcal{R}}^{p r}$ for $D L-L i t e_{\mathcal{R}}^{p r} \mathrm{KBs} \mathcal{K}$ under all the eight MBAs.

\subsection{Capturing Atom-Based Evolution}

We first study evolution under $\mathcal{L}_{\subset}^{a}$. Let $\mathcal{I}$ be an interpretation. An alignment $\operatorname{Align}(\mathcal{I}, \mathcal{N})$ of $\mathcal{I}$ with an $\overline{\operatorname{ABox}} \mathcal{N}$, is the interpretation $\{f \mid f \in$ $\mathcal{I}$ and $f$ is satisfiable with $\mathcal{N}\}$.

Consider an algorithm AlignAlg (see Algorithm 1) that inputs an evolution setting $\mathcal{K}, \mathcal{N}$, and returns the alignment $\operatorname{Align}\left(\mathcal{I}^{\text {can }}, \mathcal{N}\right)$ of a canonical model $\mathcal{I}^{\text {can }}$ of $\mathcal{K}$ : it drops all the assertions of $f c l_{\mathcal{T}}(\mathcal{A})$ contradicting $\mathcal{N}$ and keeps the rest. Using AlignAlg we can compute representation of $\mathcal{K} \diamond \mathcal{N}$ in $D L$-Lite pr $_{\mathcal{R}}^{\text {r }}$ :

Theorem 2. Let $\mathcal{K}=(\mathcal{T}, \mathcal{A})$ and $\mathcal{N}$ be an evolution setting, and $\mathcal{T}$ be in DL-Lite pr $_{\mathcal{R}}$. Then there exists a $D L$-Lite $\mathcal{R}_{\mathcal{R}}^{p r}$ representation of $\mathcal{K} \diamond \mathcal{N}$ under $\mathcal{L}_{\subseteq}^{a}$, and it can be computed in time polynomial in $|\mathcal{K} \cup \mathcal{N}|$ as follows:

$$
\mathcal{K} \diamond \mathcal{N}=\operatorname{Mod}(\mathcal{T}, \operatorname{Align} \operatorname{Alg}(\mathcal{K}, \mathcal{N}) \cup \mathcal{N}) .
$$

Example 3. Consider $\mathcal{T}=\left\{B_{0} \sqsubseteq B, B \sqsubseteq \neg C\right\}, \mathcal{A}=\{C(a)\}$, and $\mathcal{N}=B(a)$. Then, $f c l_{\mathcal{T}}(\mathcal{A})=\left\{C(a), \neg B_{0}(a), \neg B(a)\right\}$ and AlignAlg $((\mathcal{T}, \mathcal{A}), \mathcal{N})=\left\{\neg B_{0}(a)\right\}$. Hence, the result of evolution $(\mathcal{T}, \mathcal{A}) \diamond \mathcal{N}$ under $\mathcal{L}_{\subseteq}^{a}$ is $\left(\mathcal{T},\left\{B(a), \neg B_{0}(a)\right\}\right)$.

Relationships between Atom-Based Semantics. Next theorem shows that in DL-Lite $e_{\mathcal{R}}^{p r}$ all four atom-based MBAs coincide We depict these relations between semantics in Figure 2 using dashed arrows, e.g., as between $\mathcal{L}_{\subseteq}^{a}$ and $\mathcal{G}_{\#}^{a}$. Note that there is a path with solid or dashed arrows (a sequence of such arrows) between any two semantics if and only if in $D L$-Lite $e_{\mathcal{R}}^{p r}$ there is a subsumption between them. 
Theorem 4. For DL-Lite ${ }_{\mathcal{R}}^{p r}: \mathcal{L}_{\#}^{a} \equiv_{s e m} \mathcal{L}_{\subseteq}^{a} \equiv_{s e m} \mathcal{G}_{\#}^{a} \equiv_{s e m} \mathcal{G}_{\subseteq}^{a}$.

Theorems 2 and 4 imply that in DL-Lite $e_{\mathcal{R}}^{p r}$ one can use AlignAlg to compute (a representation of) evolution under all MBAs on atoms.

\subsection{Capturing Symbol-Based Evolution}

Observe that symbol-based semantics behave differently from atom-based ones: two local semantics (on set inclusion and cardinality) coincide, as well as two global semantics, while there is no subsumption between local and global ones, as depicted in Figure 2,

Theorem 5. The following relations on symbols-based MBAs hold for DL-Lite ${ }_{\mathcal{R}}^{p r}$ :

(i) $\mathcal{L}_{\subset}^{a} \preccurlyeq$ sem $\mathcal{G}_{\#}^{s}$, while $\mathcal{G}_{\#}^{s} \aleph_{\text {sem }} \mathcal{L}_{\subseteq}^{a}$;

(ii) $\mathcal{L}_{\subseteq}^{\bar{s}} \equiv_{\text {sem }} \mathcal{L}_{\#}^{s}$, and $\mathcal{G}_{\subseteq}^{s} \equiv_{\text {sem }} \mathcal{G}_{\#}^{s}$, while $\mathcal{L}_{\subseteq}^{s} \aleph_{\text {sem }} \mathcal{G}_{\#}^{s}$.

As a corollary of Theorem 5, in general the approach presented in Theorem 2 does not work for computing $\mathcal{K} \diamond \mathcal{N}$ under any of the symbol-based MBAs. At the same time, as follows from the following Theorems 6 and 8 , this approach gives complete approximations of all symbol-based semantics, while it approximates global semantics better than the local ones.

Consider the algorithm SymAlg in Algorithm 2 that will be used for evolutions on symbols. It works as follows: it inputs an evolution setting $(\mathcal{T}, \mathcal{A}), \mathcal{N}$ and a unary property $\Pi$ of assertions. Then for every atom $\phi$ in $\mathcal{N}$ it checks whether $\phi$ satisfies $\Pi$ (Line 4). If it the case, SymAlg deletes from $\operatorname{AlignAlg}((\mathcal{T}, \mathcal{A}), \mathcal{N})$ all literals $\phi^{\prime}$ that share concept name with $\phi$. Both local and global semantics have their own $\Pi: \Pi_{\mathcal{G}}$ and $\Pi_{\mathcal{L}}$.

Capturing Global Semantics. $\Pi_{\mathcal{G}}(\phi)$ checks whether $\phi$ of $\mathcal{N} \mathcal{T}$-contradicts $\mathcal{A}$ : $\Pi_{\mathcal{G}}(\phi)$ is true iff $\neg \phi \in f c l_{\mathcal{T}}(\mathcal{A}) \backslash \operatorname{Align} \operatorname{Alg}((\mathcal{T}, \mathcal{A}), \mathcal{N})$. Intuitively, SymAlg for global semantics works as follows: having contradiction between $\mathcal{N}$ and $\mathcal{A}$ on $\phi=B(c)$, the change of $B$ 's interpretation is inevitable. Since the semantics traces changes on symbols only, and $B$ is already changed, one can drop from $\mathcal{A}$ all the assertions of the form $B(d)$. Clearly, $\operatorname{Sym} \operatorname{Alg}\left(\mathcal{K}, \mathcal{N}, \Pi_{\mathcal{G}}\right)$ can be computed in time polynomial in $|\mathcal{K} \cup \mathcal{N}|$. The following theorem shows correctness of this algorithm.

Theorem 6. Let $\mathcal{K}=(\mathcal{T}, \mathcal{A})$ and $\mathcal{N}$ be an evolution setting, and $\mathcal{T}$ be in DL-Lite $\mathcal{R}^{p r}$. Then a DL-Lite ${ }_{\mathcal{R}}^{\text {pr }}$ representation of $\mathcal{K} \diamond \mathcal{N}$ under both $\mathcal{G}_{\subseteq}^{s}$ and $\mathcal{G}_{\#}^{s}$ exists and can be computed in time polynomial in $|\mathcal{K} \cup \mathcal{N}|$ as follows:

$$
\mathcal{K} \diamond \mathcal{N}=\operatorname{Mod}\left(\mathcal{T}, \operatorname{Sym} \operatorname{Alg}\left(\mathcal{K}, \mathcal{N}, \Pi_{\mathcal{G}}\right)\right)
$$

Capturing Local Semantics. Observe that $\mathcal{L}_{\subseteq}^{s}$ and $\mathcal{L}_{\#}^{s}$ are not expressible in DL-Lite $e_{\mathcal{R}}^{p r}$ because they require for a disjunction which is not available in $D$ L-Lite $\mathcal{R}_{\mathcal{R}}$ (we omit details due to space limit).

Theorem 7. DL-Lite $\mathcal{R}_{\mathcal{R}}^{p r}$ is not closed under $\mathcal{L}_{\subseteq}^{s}$ and $\mathcal{L}_{\#}^{s}$ semantics. 


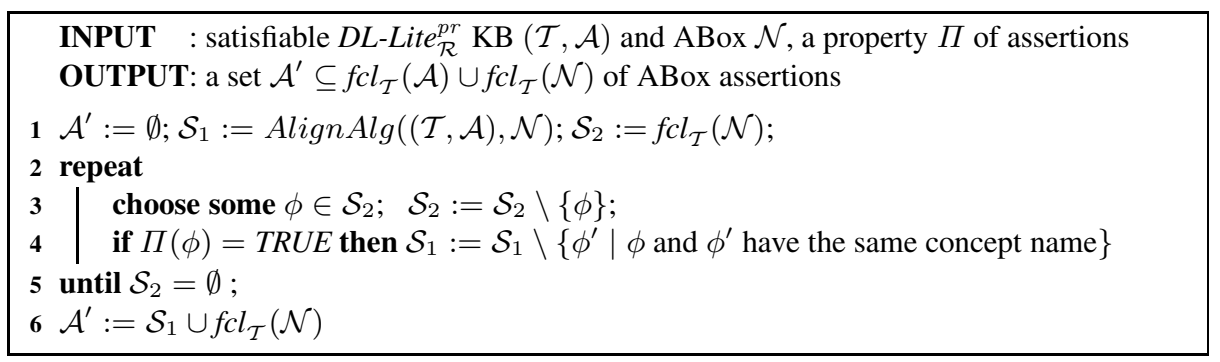

Algorithm 2. Algorithm $\operatorname{Sym} \operatorname{Alg}((\mathcal{T}, \mathcal{A}), \mathcal{N}, \Pi)$ for deterministic computation of $\mathcal{K} \diamond \mathcal{N}$ under $\mathcal{G}_{\subseteq}^{s}$ and $\mathcal{G}_{\#}^{s}$ semantics and minimal sound approximation under $\mathcal{L}_{\subseteq}^{s}$ and $\mathcal{L}_{\#}^{s}$ semantics

To compute a minimal sound approximations under local semantics on symbols, we use SymAlg with the following $\Pi_{\mathcal{L}}: \Pi_{\mathcal{L}}(\phi)$ is true iff $\phi \notin \mathcal{S}_{1}$. That is, $\Pi_{\mathcal{L}}$ checks whether the ABox $\mathcal{A} \mathcal{T}$-entails $A(c) \in f_{c} l_{\mathcal{T}}(\mathcal{N})$, and if it does not, then the algorithm deletes all the assertions from $f c l_{\mathcal{T}}(\mathcal{A})$ that share the concept name with $A(c)$. This property is weaker than the one for global semantics, since it is easier to get changes in interpretation of $A$ by choosing a model of $\mathcal{K}$ which does not include $A(c)$. The following theorem shows correctness and complexity of the algorithm.

Theorem 8. Let $\mathcal{K}=(\mathcal{T}, \mathcal{A})$ and $\mathcal{N}$ be an evolution setting, and $\mathcal{T}$ be in DL-Lite $\mathcal{R}_{\mathcal{R}}^{\text {r }}$.

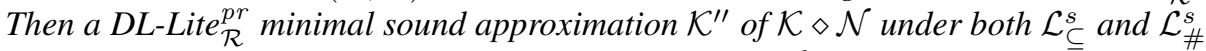
exists and can be computed in time polynomial in $|\mathcal{K} \cup \mathcal{N}|$ as follows:

$$
\mathcal{K}^{\prime \prime}=\left(\mathcal{T}, \operatorname{Sym} \operatorname{Alg}\left(\mathcal{K}, \mathcal{N}, \Pi_{\mathcal{L}}\right)\right)
$$

Example 9. Consider the following $D L$-Lite $e_{\mathcal{R}}^{p r} \operatorname{KB} \mathcal{K}=(\emptyset, \mathcal{A})$ and $\mathcal{N}$ :

$$
\mathcal{A}=\{A(a), A(b), B(c), B(d)\} ; \quad \mathcal{N}=\{\neg A(a), B(e)\} .
$$

It is easy to see that $\mathcal{A}^{\prime}=\operatorname{Sym} A \operatorname{sg}\left(\mathcal{K}, \mathcal{N}, \Pi_{\mathcal{G}}\right)$ is $\{\neg A(a), B(c), B(d), B(e)\}$, and $\mathcal{A}^{\prime \prime}=\operatorname{Sym} \operatorname{Alg}\left(\mathcal{K}, \mathcal{N}, \Pi_{\mathcal{L}}\right)$ is $\{\neg A(a), B(e)\}$. That is, $\mathcal{K} \diamond \mathcal{N}$ under $\mathcal{G}_{\beta}^{s}$ is equal to $\operatorname{Mod}\left(\emptyset, \mathcal{A}^{\prime}\right)$, and under $\mathcal{L}_{\beta}^{s}$ is approximated by $\operatorname{Mod}\left(\emptyset, \mathcal{A}^{\prime \prime}\right)$, where $\beta \in\{\subseteq, \#\}$. A closer look at $\mathcal{A}^{\prime}$ shows that the behaviour of the evolution under $\mathcal{G}_{\beta}^{s}$ is very counterintuitive: as soon as we declare that the object $a$ is not in $A$, all the information about another objects in $A$ is erased. Local semantics $\mathcal{L}_{\beta}^{s}$ are even worse: the evolution under them erases information about $B$ as soon as we just add information about a new object $e$ in $B$.

To sum up on $D L-L i t e_{\mathcal{R}}^{p r}$ : atom-based approaches (which all coincide) can be captured using a polynomial-time time algorithm based on alignment. Moreover, the evolution results produced under these MBAs are intuitive and expected, e.g., see Example 3. while symbol-based approaches produce quite unexpected and counterintuitive results (these semantics delete too much data). Furthermore, two out of four of the latter approaches cannot be captured in $D L-L i t e_{\mathcal{R}}^{p r}$. Based on these results we conclude that using atom-based approaches for applications seem to be more practical. In Figure 2 we framed in a dashed rectangle six out of eight MBAs under which DL-Lite pr $_{\mathcal{R}}$ is closed. 


\section{$5 \quad \mathcal{L}_{\subseteq}^{a}$ Evolution of DL-Lite L $_{\mathcal{R}}$ KBs}

In the previous section we showed that atom-based MBAs behave well for DL-Lite ${ }_{\mathcal{R}}^{p r}$ evolution settings, while symbol-based ones do not. This suggests to investigate atombased MBAs for the entire $D L$-Lite $\mathcal{R}_{\mathcal{R}}$. Moreover, one of the atom-based semantics $\mathcal{L}_{\subset}^{a}$ which is essentially the same as a so-called Winslett's semantics [15] (WS) was widely studied in the literature [68]. Liu, Lutz, Milicic, and Wolter studied WS for expressive DLs [6], and KBs with empty TBoxes. Most of the DLs they considered are not closed under WS. Poggi, Lembo, De Giacomo, Lenzerini, and Rosati applied WS to the same setting as we have in this work: to what they called instance level (ABox) update for $D L$ Lite [8]. They proposed an algorithm to compute the result of updates, which has technical issues, i.e., it is neither sound, nor complete [10]. They further use this algorithm to compute approximations of ABox updates in sublogics of DL-Lite, which inherits these technical issues. Actually, ABox update algorithm cannot exist since Calvanese, Kharlamov, Nutt, and Zheleznyakov showed that DL-Lite is not closed under $\mathcal{L}_{\subseteq}^{a}[11]$. We now investigate $\mathcal{L}_{\subseteq}^{a}$ evolution for $D L$-Lite $\mathcal{R}_{\mathcal{R}}$ and firstly explain why DL-Lite $\mathcal{R}_{\mathcal{R}}$ is not closed under $\mathcal{L}_{\subseteq}^{a}$.

\subsection{Understanding Inexpressibility of Evolution in DL-Lite P $_{\mathcal{R}}$}

Recall that for every $D L$-Lite $\mathrm{KB} \mathcal{K}$, the set $\operatorname{Mod}(\mathcal{K})$ has a canonical model. The following example illustrates the lack of canonical models for $\mathcal{K} \diamond \mathcal{N}$ under $\mathcal{L}_{\subseteq}^{a}$, which yields inexpressibility of $\mathcal{K} \diamond \mathcal{N}$ in $D L$-Lite.

Example 10. Consider the following DL-Lite $\mathrm{KB} \mathcal{K}_{1}=\left(\mathcal{T}_{1}, \mathcal{A}_{1}\right)$ and $\mathcal{N}_{1}=\{C(b)\}$ :

$$
\mathcal{T}_{1}=\left\{A \sqsubseteq \exists R, \exists R^{-} \sqsubseteq \neg C\right\} ; \quad \mathcal{A}_{1}=\{A(a), C(e), C(d), R(a, b)\} .
$$

Consider a model $\mathcal{I}$ of $\mathcal{K}_{1}: A^{\mathcal{I}}=\{a, x\}, C^{\mathcal{I}}=\{d, e\}$, and $R^{\mathcal{I}}=\{(a, b),(x, b)\}$, where $x \in \Delta \backslash \operatorname{adom}\left(\mathcal{K}_{1} \cup \mathcal{N}_{1}\right)$. The following models belong to $\mathcal{I} \diamond \mathcal{N}_{1}$ :

$$
\begin{array}{llll}
\mathcal{J}_{0}: & A^{\mathcal{I}}=\emptyset, & C^{\mathcal{I}}=\{d, e, b\}, & R^{\mathcal{I}}=\emptyset, \\
\mathcal{J}_{1}: & A^{\mathcal{I}}=\{x\}, & C^{\mathcal{I}}=\{e, b\}, & R^{\mathcal{I}}=\{(x, d)\}, \\
\mathcal{J}_{2}: & A^{\mathcal{I}}=\{x\}, & C^{\mathcal{I}}=\{d, b\}, & R^{\mathcal{I}}=\{(x, e)\} .
\end{array}
$$

Indeed, all the models satisfy $\mathcal{N}_{1}$ and $\mathcal{T}_{1}$. To see that they are in $\mathcal{I} \diamond \mathcal{N}_{1}$ observe that every model $\mathcal{J}(I) \in\left(\mathcal{I} \diamond \mathcal{N}_{1}\right)$ can be obtained from $\mathcal{I}$ by making modifications that guarantee that $\mathcal{J}(I) \models\left(\mathcal{N}_{1} \cup \mathcal{T}_{1}\right)$ and that the distance between $\mathcal{I}$ and $\mathcal{J}(I)$ is minimal. What are these modifications? Since in every $\mathcal{J}(I)$ the new assertion $C(b)$ holds and $\left(\exists R^{-} \sqsubseteq \neg C\right) \in \mathcal{T}_{1}$, there should be no $R$-atoms with $b$-fillers (at the second coordinate) in $\mathcal{J}(\mathcal{I})$. Hence, the necessary modifications of $\mathcal{I}$ are either to drop (some of) the $R$ atoms $R(a, b)$ and $R(x, b)$, or to modify (some of) them, by substituting the $b$-fillers with another ones, while keeping the elements $a$ and $x$ on the first coordinate. The model $\mathcal{J}_{0}$ corresponds to the case when both $R$-atoms are dropped, while in $\mathcal{J}_{1}$ and $\mathcal{J}_{2}$ only $R(a, b)$ is dropped and $R(x, b)$ is modified to $R(x, d)$ and $R(x, e)$, respectively. Note that the modification in $R(x, b)$ leads to a further change in the interpretation of $C$ in both $\mathcal{J}_{1}$ and $\mathcal{J}_{2}$, namely, $C(d)$ and $C(e)$ should be dropped, respectively.

One can verify that any model $\mathcal{J}_{\text {can }}$ that can be homomorphically embedded into $\mathcal{J}_{0}$, $\mathcal{J}_{1}$, and $\mathcal{J}_{2}$ is such that $A^{\mathcal{J}_{\text {can }}}=R^{\mathcal{J}_{\text {can }}}=\emptyset$, and $e, d \notin C^{\mathcal{J}_{\text {can }}}$. It is easy to check that 
such a model does not belong to $\mathcal{K}_{1} \diamond \mathcal{N}_{1}$. Hence, there is no canonical model in $\mathcal{K}_{1} \diamond \mathcal{N}_{1}$ and it is inexpressible in DL-Lite.

We now give an intuition why in $\mathcal{K} \diamond \mathcal{N}$ under $\mathcal{L}_{\subset}^{a}$ canonical models may be missing. Observe that in Example 10, the role $R$ is affected by the old TBox $\mathcal{T}_{1}$ as follows:

(i) $\mathcal{T}_{1}$ places (i.e., enforces the existence of) $R$-atoms in the evolution result, and on one of coordinates of these $R$-atoms, there are constants from specific sets, e.g., $A \sqsubseteq \exists R$ of $\mathcal{T}_{1}$ enforces $R$-atoms with constants from $A$ on the first coordinate, and

(ii) $\mathcal{T}_{1}$ forbids $R$-atoms in $\mathcal{K}_{1} \diamond \mathcal{N}_{1}$ with specific constants on the other coordinate, e.g., $\exists R^{-} \sqsubseteq \neg C$ forbids $R$-atoms with $C$-constants on the second coordinate.

Due to this dual-affection (both positive and negative) of the role $R$ in $\mathcal{T}_{1}$, we were able to provide ABoxes $\mathcal{A}_{1}$ and $\mathcal{N}_{1}$, which together triggered the case analyses of modifications on the model $\mathcal{I}$, that is, $\mathcal{A}_{1}$ and $\mathcal{N}_{1}$ were triggers for $R$. Existence of such an affected $R$ and triggers $\mathcal{A}_{1}$ and $\mathcal{N}_{1}$ made $\mathcal{K}_{1} \diamond \mathcal{N}_{1}$ inexpressible in DL-Lite $\mathcal{R}_{\mathcal{R}}$. Therefore, we now formally define and then learn how to detect dually-affected roles in TBoxes $\mathcal{T}$ and how to understand whether these roles are triggered by $\mathcal{A}$ and $\mathcal{N}$.

Definition 11. Let $\mathcal{T}$ be a DL-Lite $\mathcal{R}_{\mathcal{R}}$ TBox. Then a role $R$ is dually-affected in $\mathcal{T}$ if for some concepts $A$ and $B$ it holds that $\mathcal{T} \models A \sqsubseteq \exists R$ and $\mathcal{T} \models \exists R^{-} \sqsubseteq \neg B$. A duallyaffected role $R$ is triggered by $\mathcal{N}$ if there is a concept $C$ such that $\mathcal{T} \models \exists R^{-} \sqsubseteq \neg C$ and $\mathcal{N} \models_{\mathcal{T}} C(b)$ for some constant $b$.

As we saw in Example 10, even one dually-affected role in a TBox can cause inexpressibility of evolution. Moreover, if there is a dually affected role, we can always find $\mathcal{A}$ and $\mathcal{N}$ to trigger it. We generalize this observation as follows:

Theorem 12. Let $\mathcal{T}$ be a DL-Lite $\mathcal{R}_{\mathcal{R}}$ TBox and $R$ be a role dually affected in $\mathcal{T}$. Then there exist ABoxes $\mathcal{A}$ and $\mathcal{N}$ s.t. $(\mathcal{T}, \mathcal{A}) \diamond \mathcal{N}$ is inexpressible in $D L$-Lite $\mathcal{R}$ under $\mathcal{L}_{\subseteq}^{a}$.

\subsection{Prototypes}

Closer look at the sets of models $\mathcal{K} \diamond \mathcal{N}$ for $D L$-Lite $\mathcal{R}$ KBs $\mathcal{K}$ gives a surprising result:

Theorem 13. The set of models $\mathcal{K} \diamond \mathcal{N}$ under $\mathcal{L}_{\subseteq}^{a}$ can be divided (but in general not partitioned) into worst-case exponentially many in $|\mathcal{K} \cup \mathcal{N}|$ subsets $\mathcal{S}_{0}, \ldots, \mathcal{S}_{n}$, where each $\mathcal{S}_{i}$ has a canonical model $\mathcal{J}_{i}$, which is a minimal element in $\mathcal{K} \diamond \mathcal{N}$ wrt homomorphisms.

We call these $\mathcal{J}_{i}$ s prototypes. Thus, capturing $\mathcal{K} \diamond \mathcal{N}$ in some logics boils down to (i) capturing each $\mathcal{S}_{i}$ with some theory $\mathcal{K}_{\mathcal{S}_{i}}$ and (ii) taking the disjunction across all $\mathcal{K}_{\mathcal{S}_{i}}$. This will give the desired theory $\mathcal{K}^{\prime}=\mathcal{K}_{\mathcal{S}_{1}} \vee \cdots \vee \mathcal{K}_{\mathcal{S}_{n}}$ that captures $\mathcal{K} \diamond \mathcal{N}$. As we will see some of $\mathcal{K}_{\mathcal{S}_{i}}$ s are not $D L$-Lite theories (while they are $\mathcal{S H O I Q}$ theories, see Section 5.4 for details). We construct each $\mathcal{K}_{\mathcal{S}_{i}}$ in two steps. First, we construct a $D L$-Lite $\mathcal{R}_{\mathcal{R}} \mathrm{KB} \mathcal{K}\left(\mathcal{J}_{i}\right)$ which is a sound approximations of $\mathcal{S}_{i}$, i.e., $\mathcal{S}_{i} \subseteq \operatorname{Mod}\left(\mathcal{K}\left(\mathcal{J}_{i}\right)\right)$. Second, based on $\mathcal{K}$ and $\mathcal{N}$, we construct a $\mathcal{S H O I Q}$ formula $\Psi$, which cancels out all the models in $\operatorname{Mod}\left(\mathcal{K}\left(\mathcal{J}_{i}\right)\right) \backslash \mathcal{S}_{i}$, i.e., $\mathcal{K}_{\mathcal{S}_{i}}=\Psi \wedge \mathcal{K}\left(\mathcal{J}_{i}\right)$. Finally,

$\mathcal{K}_{\mathcal{S}_{0}} \vee \cdots \vee \mathcal{K}_{\mathcal{S}_{n}}=\left(\Psi \wedge \mathcal{K}\left(\mathcal{J}_{0}\right)\right) \vee \cdots \vee\left(\Psi \wedge \mathcal{K}\left(\mathcal{J}_{n}\right)\right)=\Psi \wedge\left(\mathcal{K}\left(\mathcal{J}_{0}\right) \vee \cdots \vee \mathcal{K}\left(\mathcal{J}_{n}\right)\right)$ 

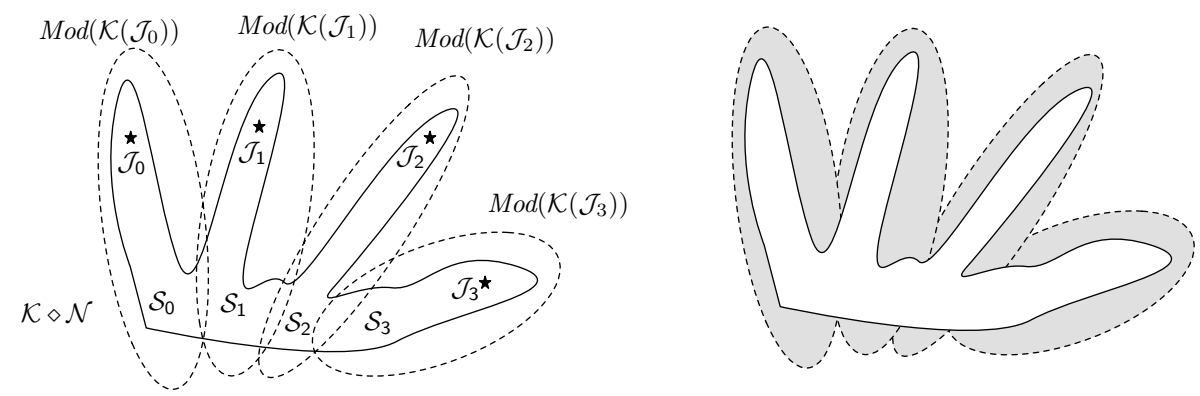

Fig. 3. Graphical representation of our approach to capture the result of evolution under $\mathcal{L}_{\subseteq}^{a}$

To get a better intuition on our approach consider Figure 3 , where the result of evolution $\mathcal{K} \diamond \mathcal{N}$ is depicted as the figure with solid-line borders (each point within the figure is a model of $\mathcal{K} \diamond \mathcal{N})$. For the sake of example, let $\mathcal{K} \diamond \mathcal{N}$ under $\mathcal{L}_{\subset}^{a}$ can be divided in four subsets $\mathcal{S}_{0}, \ldots, \mathcal{S}_{3}$. To emphasize this fact, $\mathcal{K} \diamond \mathcal{N}$ looks similar to a hand with four fingers, where each finger represents an $\mathcal{S}_{i}$. Consider the left part of Figure 3 , where the canonical $\mathcal{J}_{i}$ model of each $\mathcal{S}_{i}$ is depicted as a star. Using $D L$-Lite $e_{\mathcal{R}}$, we can provide KBs $\mathcal{K}\left(\mathcal{J}_{i}\right)$ s that are sound approximation of corresponding $\mathcal{S}_{i}$ s. We depict the models $\operatorname{Mod}\left(\mathcal{K}\left(\mathcal{J}_{i}\right)\right)$ as ovals with dashed-line boarders. In the right part of Figure 3 we depict in grey the models $\operatorname{Mod}\left(\mathcal{K}\left(\mathcal{J}_{i}\right)\right) \backslash \mathcal{S}_{i}$ that are cut off by $\Psi$.

We now define prototypes formally and proceed to procedures discussed above.

Definition 14. Let $\mathcal{K}$ and $\mathcal{N}$ be an evolution setting. A prototypal set for $\mathcal{K} \diamond \mathcal{N}$ un$\operatorname{der} \mathcal{L}_{\subseteq}^{a}$ is a minimal subset $=\left\{\mathcal{J}_{0}, \ldots, \mathcal{J}_{n}\right\}$ of $\mathcal{K} \diamond \mathcal{N}$ satisfying the following property:

for every $\mathcal{J} \in \mathcal{K} \diamond \mathcal{N}$ there exists $\mathcal{J}_{i} \in$ homomorphically embeddable in $\mathcal{J}$.

We call every $\mathcal{J}_{i} \in$ a prototype for $\mathcal{K} \diamond \mathcal{N}$. Note that prototypes generalize canonical models in the sense that every set of models with a canonical one, say $\operatorname{Mod}(\mathcal{K})$ for a $D L-$ Lite $_{\mathcal{R}} \mathrm{KB} \mathcal{K}$, has a prototype, which is exactly this canonical model.

\subsection{Computing $\mathcal{L}_{\subseteq}^{a}$ Evolution for DL-Lite L $_{\mathcal{R}}$}

For the ease of exhibition of our procedure that computes evolution $\mathcal{K} \diamond \mathcal{N}$ under $\mathcal{L}_{\subseteq}^{a}$ semantics we restrict $D L$-Lite $_{\mathcal{R}}$ by assuming that TBoxes $\mathcal{T}$ should satisfy: for any two roles $R$ and $R^{\prime}, \mathcal{T} \not \forall \exists R \sqsubseteq \exists R^{\prime}$ and $\mathcal{T} \not \forall \exists R \sqsubseteq \neg \exists R^{\prime}$. That is, we forbid direct interaction (subsumption and disjoint) between role projections and call such $\mathcal{T}$ as without direct role interactions. Some interaction between $R$ and $R^{\prime}$ is still possible, e.g., role projections may contain the same concept. This restriction allows us to analyze evolution that affects roles independently for every role. We will further comment on how the following techniques can be extended to the case when roles interact in an arbitrary way.

Components for Computation. We now introduce several notions and notations that we further use in the description of our procedure. The notion of alignment was introduced 
$\operatorname{BZP}(\mathcal{K}, \mathcal{N})$

1. $\mathcal{J}_{0}:=\operatorname{Align}\left(\mathcal{I}^{\text {can }}, \mathcal{N}\right) \cup \mathcal{N}$, where $\mathcal{I}^{\text {can }}$ is the canonical model of $\mathcal{K}$.

2. For each $R(a, b) \in A A(\mathcal{K}, \mathcal{N})$, do $\mathcal{J}_{0}:=\mathcal{J}_{0} \backslash\{R(a, b)\}$,

if there is no $R(a, \beta) \in \mathcal{I}^{\text {can }} \backslash A A(\mathcal{K}, \mathcal{N})$ do $\mathcal{J}_{0}:=\mathcal{J}_{0} \backslash \operatorname{root}_{\mathcal{T}}^{a t}(\exists R(a))$.

3. Return $\mathcal{J}_{0}$.

Fig. 4. The procedure of building zero-prototype

in Section 4.1 An auxiliary set of atoms $A A$ (Auxiliary Atoms) that, due to evolution, should be deleted from the original $\mathrm{KB}$ and have some extra condition on the first coordinate is:

$A A(\mathcal{T}, \mathcal{A}, \mathcal{N})=\left\{R(a, b) \in f_{c} l_{\mathcal{T}}(\mathcal{A}) \mid \mathcal{T} \models A \sqsubseteq \exists R, \mathcal{A} \models_{\mathcal{T}} A(a), \mathcal{N} \models_{\mathcal{T}} \neg \exists R^{-}(b)\right\}$.

If $R_{i}$ is a dually-affected role of $\mathcal{T}$ triggered by $\mathcal{A}$ and $\mathcal{N}$, then the set of forbidden atoms (of the original $\mathrm{ABox}$ ) $\mathrm{FA}[\mathcal{T}, \mathcal{A}, \mathcal{N}]\left(R_{i}\right)$ for $R_{i}$ is:

$$
\left\{D(c) \in f c l_{\mathcal{T}}(\mathcal{A}) \mid \exists R_{i}^{-}(c) \wedge D(c) \models_{\mathcal{T}} \perp \text { and } \mathcal{N} \forall_{\mathcal{T}} D(c) \text {, and } \mathcal{N} \forall_{\mathcal{T}} \neg D(c)\right\} .
$$

Consequently, the set of forbidden atoms for the entire $\operatorname{KB}(\mathcal{T}, \mathcal{A})$ and $\mathcal{N}$ is

$$
\mathrm{FA}(\mathcal{T}, \mathcal{A}, \mathcal{N})=\bigcup_{R_{i} \in T R} \operatorname{FA}(\mathcal{T}, \mathcal{A}, \mathcal{N})\left(R_{i}\right)
$$

where $T R(\mathcal{T}, \mathcal{N})$ (or simply $T R$, which stands for triggered roles) is the set of all roles dually-affected in $\mathcal{T}$ that are triggered by $\mathcal{N}$. In the following we omit the arguments $(\mathcal{T}, \mathcal{A}, \mathcal{N})$ in FA when they are clear from the context. For a role $R$, the set $S C(R)$, where $S C$ stands for sub-concepts, is a set of concepts that are immediately under $\exists R$ in the concept hierarchy generated by $\mathcal{T}$ :

$S C(R)=\left\{A \mid \mathcal{T} \models A \sqsubseteq \exists R\right.$ and there is no $A^{\prime}$ s.t. $\mathcal{T} \models A \sqsubseteq A^{\prime}$ and $\left.\mathcal{T} \models A^{\prime} \sqsubseteq \exists R\right\}$.

If $f$ is an ABox assertion, then $\operatorname{root}_{\mathcal{T}}^{a t}(f)$ is a set of all the atoms that $\mathcal{T}$-entail $f$. For example, if $\mathcal{T} \models A \sqsubseteq \exists R$, then $A(x) \in \operatorname{root}_{\mathcal{T}}^{a t}(\exists R(x))$.

We are ready to proceed to construction of prototypes.

Constructing Zero-Prototype. The procedure $B Z P(\mathcal{K}, \mathcal{N})$ (Build Zero Prototype) in Figure 4 constructs the main prototype $\mathcal{J}_{0}$ for $\mathcal{K}$ and $\mathcal{N}$, which we call zero-prototype. Based on $\mathcal{J}_{0}$ we will construct all the other prototypes. To build $\mathcal{J}_{0}$ one should align the canonical model $\mathcal{I}^{\text {can }}$ of $\mathcal{K}$ with $\mathcal{N}$, and then delete from the resulting set of atoms all the auxiliary atoms $R(a, b)$ of $A A(\mathcal{K}, \mathcal{N})$. If $\mathcal{I}^{\text {can }}$ contains no atoms $R(a, \beta) \in A A(\mathcal{K}, \mathcal{N})$ for some $\beta$, then we further delete atoms $\operatorname{root}_{\mathcal{T}}^{a t}(\exists R(a))$ from $\mathcal{J}_{0}$, otherwise would we get a contradiction with the TBox. Note that $\mathcal{J}_{0}$ can be infinite.

Constructing Other Prototypes. The procedure $B P\left(\mathcal{K}, \mathcal{N}, \mathcal{J}_{0}\right)$ (Build Prototypes) of constructing takes $\mathcal{J}_{0}$ and, based on it, builds the other prototypes by (i) dropping FA-atoms from $\mathcal{J}_{0}$ and then (ii) adding atoms necessary to obtain a model of $\mathcal{K} \diamond \mathcal{N}$. This procedure can be found in Figure 5

We conclude the discussion on the procedures with a theorem: 
1. $:=\left\{\mathcal{J}_{0}\right\}$.

2. For each subset $\mathcal{D}=\left\{D_{1}\left(c_{1}\right), \ldots, D_{k}\left(c_{k}\right)\right\} \subseteq$ FA do for each $\mathcal{R}=\left(R_{i_{1}}, \ldots, R_{i_{k}}\right)$ such that $D_{j}\left(c_{j}\right) \in \mathrm{FA}\left(R_{i_{j}}\right)$ for $j=1, \ldots, k$ do for each $\mathcal{B}=\left(A_{i_{1}}, \ldots, A_{i_{k}}\right)$ such that $A_{j} \in S C\left(R_{j}\right)$ do $\mathcal{J}[\mathcal{D}, \mathcal{R}, \mathcal{B}]:=\left[\mathcal{J}_{0} \backslash \bigcup_{i=1}^{k} \operatorname{root}_{\mathcal{T}}\left(D_{i}\left(c_{i}\right)\right)\right] \cup \bigcup_{j=1}^{k}\left[f l_{\mathcal{T}}\left(R_{i_{j}}\left(x_{j}, c_{j}\right)\right) \cup\left\{A_{R_{i_{j}}}\left(x_{j}\right)\right\}\right]$, where all $x_{i}$ 's are different constants from $\Delta \backslash \operatorname{adom}(\mathcal{K})$, fresh for $\mathcal{I}^{\text {can }}$. $:=\cup\{\mathcal{J}[\mathcal{D}, \mathcal{R}, \mathcal{B}]\}$.

3. Return.

Fig. 5. The procedure of building prototypes in DL-Lite $e_{\mathcal{R}}$ without direct role interactions based on the zero prototype $\mathcal{J}_{0}$

Theorem 15. Let $\mathcal{K}=(\mathcal{T}, \mathcal{A}), \mathcal{N}$ be an evolution setting and $\mathcal{T}$ without direct role interactions. Then the set $B P(\mathcal{K}, \mathcal{N}, B Z P(\mathcal{K}, \mathcal{N}))$ is prototypal for $\mathcal{K} \diamond \mathcal{N}$ under $\mathcal{L}_{\subseteq}^{a}$. Continuing with Example 10, one can check that the prototypal set for $\mathcal{K}_{1}$ and $\mathcal{N}_{1}$ is $\left\{\mathcal{J}_{0}, \mathcal{J}_{1}, \mathcal{J}_{2}, \mathcal{J}_{3}\right\}$, where $\mathcal{J}_{0}, \mathcal{J}_{1}$, and $\mathcal{J}_{2}$ are as in the example and $A^{\mathcal{J}_{3}}=\{x, y\}$, $C^{\mathcal{J}_{3}}=\{b\}$, and $R^{\mathcal{J}_{3}}=\{(x, d),(y, e)\}$.

We proceed to correctness of BP in capturing evolution in $D L$-Lite L $_{\mathcal{R}}$, where we use the following set $\operatorname{FC}[\mathcal{T}, \mathcal{A}, \mathcal{N}]\left(R_{i}\right)=\left\{c \mid D(c) \in \mathrm{FA}[\mathcal{T}, \mathcal{A}, \mathcal{N}]\left(R_{i}\right)\right\}$, that collects all the constants that participate in the forbidden atoms.

Theorem 16. Let $\mathcal{K}=(\mathcal{T}, \mathcal{A}), \mathcal{N}$ be an evolution setting, $\mathcal{T}$ without direct role interactions, and $B P(\mathcal{K}, \mathcal{N}, B Z P(\mathcal{K}, \mathcal{N}))=\left\{\mathcal{J}_{0}, \ldots, \mathcal{J}_{n}\right\}$ a prototypal set for $\mathcal{K} \diamond \mathcal{N}$. Then $\mathcal{K} \diamond \mathcal{N}$ under $\mathcal{L}_{\subseteq}^{a}$ is expressible in $\mathcal{S H O I} \mathcal{Q}$ and moreover

$$
\mathcal{K} \diamond \mathcal{N}=\operatorname{Mod}\left(\Psi \wedge\left(\left(\mathcal{T}, \mathcal{A}_{0}\right) \vee \cdots \vee\left(\mathcal{T}, \mathcal{A}_{n}\right)\right)\right)
$$

where $A_{i}$ is a DL-Lite $\mathcal{R}_{\mathcal{R}}$ ABox such that $\mathcal{J}_{i}$ is a canonical model for $\left(\mathcal{T}, \mathcal{A}_{i}\right), \Psi=$ $\forall R_{\top} .\left(\Phi_{1} \sqcap \Phi_{2}\right)$, where $R_{\top}$ is the top role (which is present in OWL 2) and

$$
\begin{gathered}
\Phi_{1} \equiv \prod_{R_{i} \in T R} \prod_{c_{j} \in \mathrm{FC}\left[R_{i}\right]}\left[\left(\exists R_{i} \cdot\left\{c_{j}\right\} \sqsubseteq(\leq 1 R . \top)\right) \sqcap\left(\exists R_{i} \cdot\left\{c_{j}\right\} \sqsubseteq \bigsqcup_{B(x) \in \operatorname{root}_{\mathcal{T}}^{a t}\left(\exists R_{i}(x)\right)} \neg\{x\} \sqcup B\right)\right], \\
\Phi_{2} \equiv \prod_{R(a, b) \in A A}\left[\neg(\{a\} \sqsubseteq \exists R . \top) \sqcup \underset{C(a) \in \operatorname{root}_{\mathcal{T}}^{a t}(\exists R(a)) \cap f_{\mathcal{T}}(\mathcal{A})}{\{a\} \sqsubseteq C] .}\right.
\end{gathered}
$$

What is missing in the theorem above is how to compute the ABoxes $\mathcal{A}_{i}$ s. One can do it using a similar procedure to the one of constructing $\mathcal{J}_{i} \mathrm{~s}$, with the difference that one has to take the original ABox $\mathcal{A}$ instead of $\mathcal{I}^{\text {can }}$ as the input. Note that $\mathcal{A}$ may include negative atoms, like $\neg B(c)$, which should be treated in the same way as positive ones.

Continuing with Example 10 the ABoxes $\mathcal{A}_{0}$ and $\mathcal{A}_{1}$ are as follows:

$$
\mathcal{A}_{0}=\{C(d), C(e), C(b)\} ; \quad \mathcal{A}_{1}=\{A(x), C(e), C(b), R(x, d)\} .
$$


$\mathcal{A}_{2}$ and $\mathcal{A}_{3}$ can be built in the similar way. Note that only $\mathcal{A}_{0}$ is in $D L$-Lite $\mathcal{R}$, while writing $\mathcal{A}_{1}, \ldots, \mathcal{A}_{3}$ requires variables in ABoxes. Variables, also known as soft constants, are not allowed in DL-Lite $\mathcal{R}_{\mathcal{R}}$ ABoxes, while present in DL-Lite $\mathcal{R}_{\mathcal{S}}$ ABoxes. Soft constants $x$ are constants not constrained by the Unique Name Assumption: it is not necessary that $x^{\mathcal{I}}=x$. Since $D L$-Lite RS $_{\mathcal{S}}$ is tractable and first-order rewritable [12], expressing $\mathcal{A}_{1}$ in $D L$-Lite $e_{\mathcal{R} S}$ instead of $D$ L-Lite L $_{\mathcal{R}}$ does not affect tractability.

Note that the number of prototypes is exponential in the number of constants, and therefore the size of the $\mathcal{S H O I Q}$ theory described in Theorem 16 is also exponential in the number of constants.

Capturing $\mathcal{L}_{\subseteq}^{a}$ Semantics for DL-Lite $\mathcal{R}_{\mathcal{R}}$ KBs with Direct Role Interactions. In this general case the BP procedure does return prototypes but not all of them. To capture the $\mathcal{L}_{\subseteq}^{a}$ for such KBs one should iterate BP over (already constructed) prototypes until no new prototypes can be constructed. Intuitively the reason is that BP deletes forbidden atoms (atoms of FA) and add new atoms of the form $R(a, b)$ for some triggered duallyaffected role $R$ which may in turn trigger another dually-affected role, say $P$, and such triggering may require further modifications, already for $P$. This further modification require a new run of BP. For example, if we have $\exists R^{-} \sqsubseteq \neg \exists P^{-}$in the TBox and we set $R(a, b)$ in a prototype, say $\mathcal{J}_{k}$, this modification triggers role $P$ and we should run BP recursively with the prototype $J_{k}$ as if it was the zero prototype. We shall not discuss the general procedures in more details due to space limit.

\subsection{Practical Considerations on $\mathcal{L}_{\subseteq}^{a}$ Evolution}

As a summary of Sections 4 and 5 , we now discuss how one can compute $\mathcal{L}_{\subseteq}^{a}$-evolution of $D L$-Lite L $_{\mathcal{R}} \mathrm{KBs}$ in practice. For an evolution setting $\mathcal{K}$ and $\mathcal{N}$ consider the following procedure of computing $\mathcal{K}^{\prime}$ such that $\operatorname{Mod}\left(\mathcal{K}^{\prime}\right)=\mathcal{K} \diamond \mathcal{N}$ :

1. Check whether $\mathcal{K}$ is in $D L$-Lite $e_{\mathcal{R}}^{p r}$. This test can be done in polynomial time, see Section 4

2. If $\mathcal{K}$ is in $D L$-Lite $e_{\mathcal{R}}^{p r}$, then $\mathcal{K}^{\prime}$ is in $D L$-Lite $\mathcal{R}_{\mathcal{R}}^{p r}$ and can be computed in polynomial time using the algorithm AlignAlg as described in Theorem 2 of Section 4

3. If $\mathcal{K}$ is not in $D L-L i t e_{\mathcal{R}}^{p r}$, then check whether any dually-affected role of $\mathcal{K}$ is triggered by $\mathcal{N}$. This test can be done in polynomial time, see Section 5.1 .

4. If the test of Case 3 fails, then $\mathcal{K}^{\prime}$ is in $D L$-Lite $\mathcal{R}_{\mathcal{R}}$ and can be computed as in Case 2

5. If the test of Case 3 succeeds, then $\mathcal{K}^{\prime}$ is in $\mathcal{S H O I} \mathcal{Q}$, but not in DL-Lite $\mathcal{R}$, and can be computed using prototype-based techniques as described in Theorem 16 The size of this $\mathcal{K}^{\prime}$ is polynomial in the number of prototypes for $\mathcal{K} \diamond \mathcal{N}$. Since the number of prototypes is worst-case exponential in the number of constants in $\mathcal{A}$, the size of $\mathcal{K}^{\prime}$ could be exponential in $|\mathcal{K} \cup \mathcal{N}|$.

The case when computation of $\mathcal{K}^{\prime}$ can be intractable is of $\mathcal{K}$ with dually-affected roles triggered by $\mathcal{N}$. It is unclear how often this case may occur in practice. While the tractable case of $D L$-Lite $e_{\mathcal{R}}^{p r}$ where we disallow assertions of the form $\exists R \sqsubseteq \neg A$ seems to be practical, since it extends the (first-order fragment) of RDFS.

We now discuss a way to approximate $\mathcal{K} \diamond \mathcal{N}$ with a $D L$-Lite L $_{\mathcal{R}} \mathrm{KB}$ when this set is not expressible in $D L$-Lite Ret $_{\mathcal{R}}$. Let $\mathcal{K}=(\mathcal{T}, \mathcal{A})$ and $\mathcal{N}$ be an evolution setting, then a DL-Lite R $_{\mathcal{R}} \mathrm{KB} \mathcal{K}^{c}=\left(\mathcal{T}, \mathcal{A}^{c}\right)$ is a certain $\mathcal{L}_{\subseteq}^{a}$-approximation of $\mathcal{K} \diamond \mathcal{N}$ if $\mathcal{A}^{c}=\{F \mid K \diamond$ $\mathcal{N} \models F\}$. We called $\mathcal{K}^{c}$ certain since it resembles certain answers for queries over KBs. 
Proposition 17. Let $\mathcal{K}, \mathcal{N}$ be an evolution setting. Then the certain $\mathcal{L}_{\subseteq}^{a}$-approximation of $\mathcal{K} \diamond \mathcal{N}$ exists, unique, and can be computed in non-deterministic exponential time.

Proof. Clearly all ABox assertions of $\mathcal{A}^{c}$ are over concepts, roles, and constants of $\mathcal{K}$, thus, there are at most a quadratic many (in $|\mathcal{K} \cup \mathcal{N}|$ ) of them, and we can simply test whether $F \in \mathcal{A}^{c}$ for each such assertion $F$. Since $\mathcal{K} \diamond \mathcal{N}$ is representable in $\mathcal{S H O I} \mathcal{Q}$,

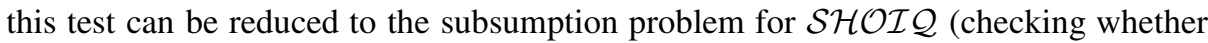
$\mathcal{K}^{\prime} \models C(a)$ is equivalent to checking whether $\left.\mathcal{K}^{\prime} \models\{a\} \sqsubseteq C\right)$. Subsumption for

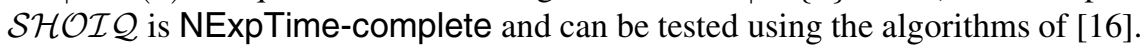

The proposition above gives the upper bound for $\mathcal{K}^{c}$ computations. We do not know the lower bound, but conjecture it to be in polynomial time. Note that NExpTime lower bound for $\mathcal{S H O I} \mathcal{Q}$ subsumption checking holds for arbitrary $\mathcal{S H O I} \mathcal{Q}$ concepts, while Theorem 16 gives us $\mathcal{K}^{\prime}$ with concepts of a specific kind. Moreover, the authors of [16] argue that despite the high complexity of subsumption checking their algorithms should behave well in many typically encountered cases. Note also that for DL-Lite $e_{\mathcal{R}}^{p r} \mathrm{KBs}$ certain approximations in fact capture the evolution result, that is $\operatorname{Mod}\left(\mathcal{K}^{c}\right)=\mathcal{K} \diamond \mathcal{N}$.

\section{Conclusion}

We studied model-based approaches to ABox evolution (update and revision) over $D L-$ Lite $_{\mathcal{R}}$ and its fragment DL-Lite $e_{\mathcal{R}}^{p r}$, which both extend (first-order fragment of) RDFS. $D L-$ Lite $_{\mathcal{R}}^{p r}$ is closed under most of the MBAs, while $D L-$ Lite $_{\mathcal{R}}$ is not closed under any of them. We showed that if the TBox of $\mathcal{K}$ entails a pair of assertions of the form $\mathcal{A} \sqsubseteq \exists R$ and $\exists R^{-} \sqsubseteq \neg C$, then an interplay of $\mathcal{N}$ and $\mathcal{A}$ may lead to inexpressibility of $\mathcal{K} \diamond \mathcal{N}$. For $D L$-Lite $e_{\mathcal{R}}^{p r}$ we provided algorithms how to compute evolution results for six model-based approaches and approximate for the remaining two. For DL-Lite $\mathcal{R}$

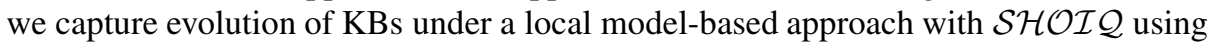
novel techniques based on what we called prototypes. We believe that prototypes are important since they can be used to study evolution for ontology languages other than $D L$-Lite $\mathcal{R}_{\mathcal{R}}$. Finally, we showed how to approximate evolution when it is not expressible in $D L-$ Lite $_{\mathcal{R}}$ using what we called certain approximations.

It is the first attempt to provide an understanding of inexpressibility of MBAs for $D L$ Lite evolution. Without this understanding it is unclear how to proceed with the study of evolution in more expressive DLs and what to expect from MBAs in such logics. We also believe that our techniques of capturing semantics based on prototypes give a better understanding of how MBAs behave.

Acknowledgements. We are thankful to Diego Calvanese, Balder ten Cate, and Werner Nutt for insightful discussions. We thank anonymous reviewers for constructive comments. The authors are supported by EU projects ACSI (FP7-ICT-257593) and Ontorule (FP7-ICT-231875); the first author is supported by ERC FP7 grant Webdam (agreement n. 226513).

\section{References}

1. Baader, F., Calvanese, D., McGuinness, D., Nardi, D., Patel-Schneider, P.F. (eds.): The Description Logic Handbook: Theory, Implementation and Applications. Cambridge University Press (2003) 
2. Flouris, G., Manakanatas, D., Kondylakis, H., Plexousakis, D., Antoniou, G.: Ontology change: Classification and survey. Knowledge Engineering Review 23(2), 117-152 (2008)

3. Abiteboul, S., Grahne, G.: Update semantics for incomplete databases. In: VLDB (1985)

4. Katsuno, H., Mendelzon, A.: On the difference between updating a knowledge base and revising it. In: Proc. of KR 1991, pp. 387-394 (1991)

5. Eiter, T., Gottlob, G.: On the complexity of propositional knowledge base revision, updates and counterfactuals. Artificial Intelligence 57, 227-270 (1992)

6. Liu, H., Lutz, C., Milicic, M., Wolter, F.: Updating description logic ABoxes. In: KR (2006)

7. Calvanese, D., Giacomo, G.D., Lembo, D., Lenzerini, M., Rosati, R.: Tractable reasoning and efficient query answering in description logics: The DL-Lite family. J. of Automated Reasoning 39(3), 385-429 (2007)

8. Giacomo, G.D., Lenzerini, M., Poggi, A., Rosati, R.: On instance-level update and erasure in description logic ontologies. J. of Logic and Computation 19(5), 745-770 (2009)

9. Wang, Z., Wang, K., Topor, R.W.: A new approach to knowledge base revision in DL-Lite. In: AAAI (2010)

10. Calvanese, D., Kharlamov, E., Nutt, W., Zheleznyakov, D.: Evolution of DL-lite Knowledge Bases. In: Patel-Schneider, P.F., Pan, Y., Hitzler, P., Mika, P., Zhang, L., Pan, J.Z., Horrocks, I., Glimm, B. (eds.) ISWC 2010, Part I. LNCS, vol. 6496, pp. 112-128. Springer, Heidelberg (2010)

11. Calvanese, D., Kharlamov, E., Nutt, W., Zheleznyakov, D.: Evolution of DL-Lite knowledge bases (extended version). Technical report, KRDB Research Centre, Free Univ. of Bolzano (2011)

12. Artale, A., Calvanese, D., Kontchakov, R., Zakharyaschev, M.: The DL-Lite family and relations. J. of Artificial Intelligence Research 36, 1-69 (2009)

13. Poggi, A., Lembo, D., Calvanese, D., Giacomo, G.D., Lenzerini, M., Rosati, R.: Linking data to ontologies. J. on Data Semantics, 133-173 (2008)

14. Calvanese, D., Kharlamov, E., Nutt, W.: A proof theory for DL-Lite. In: DL (2007)

15. Winslett, M.: Updating Logical Databases. Cambridge University Press (1990)

16. Horrocks, I., Sattler, U.: A tableau decision procedure for SHOIQ. J. Autom. Reasoning 39(3), 249-276 (2007) 